\title{
APROXIMACIONES Y RESULTADOS PARA LA MEDICIÓN PRÁCTICA DE ILUMINANCIA
}

\author{
Luis Diego Marín Naranjo
}

\begin{abstract}
Resumen
La teoría de la ley de cuadrado inverso y la relación con la medición práctica de la iluminancia se analizó en otro trabajo publicado en este mismo número de revista denominado Análisis de la ley de cuadrado inverso para medición práctica de iluminancia, por lo que en este otro artículo se realiza un estudio más detallado con simulación en computador de la iluminancia. En la primera parte se describen las aproximaciones en las ecuaciones relacionadas a la iluminancia con la distancia y los resultados obtenidos con la simulación en computador. En la segunda parte se analizan los resultados de una práctica experimental para la medición de iluminancia con un banco fotométrico, con lo que se puede verificar la ley de cuadrado inverso.
\end{abstract}

Palabras clave: fotometría, mediciones, iluminancia, ley de cuadrado inverso, metrología.

\begin{abstract}
The theory of inverse square law and the relationship with the practical measurement of luminance were analyzed in other paper published in this same issue under the name Inverse square law analysis for practical measurement of luminance so we proceed to further study using computer simulation for the luminance. The first part of this paper is a description of approximations in the equations related to luminance with the distance and results obtained in the computed simulation. The second part is a analysis of results obtained in an experimental practice to measure luminance using a photometric bench, which allows to obtain the inverse square law.
\end{abstract}

Key words: photometry, measurements, illuminance, inverse square law, metrology.

Recibido: 24 de marzo del 2009 • Aprobado: 26 de mayo del 2009.

\section{CÁlCULOS DE APROXIMACIÓN DE LEY DE CUADRADO INVERSO}

Este documento es el resultado de un informe de investigación en la Universidad de Costa Rica acerca de mediciones fotométricas y la ley de cuadrado inverso. Además se parte de información técnica de otras referencias indicadas al final.

El objetivo es medir la iluminancia $E_{v}$ en un punto $\mathrm{P}$ a diferentes distancias $D$. El diámetro de la fuente es $2 R$, lo cual puede ser la abertura en la fuente o en una pantalla con ese diámetro. Estos valores medidos son datos en la ecuación (1) en forma teórica y se supone que son los mismos valores.

$$
E_{v}=\frac{\partial R^{2} L_{v}}{D^{2}+R^{2}}
$$

Se calcula ahora $E_{v}$ con la ecuación (2) $(\beta=0$ en este caso) usando la errónea consideración de que la ley de cuadrado inverso se mantiene para la fuente entera.

$E_{v}=\frac{d \Phi_{v}}{d A^{\prime}}=\frac{I_{v} \cos \beta}{r^{2}}$

Los resultados se presentan en el Cuadro 1 para un ejemplo, considerando $R$ (radio) $=0,015$ m (lámpara de $3 \mathrm{~cm}$ de diámetro), $L_{v}=5380$ 
$\mathrm{cd} / \mathrm{m}^{2}$ y calculando $I_{v}=3,802897907 \mathrm{~cd}$ de la ecuación (3).

$$
I_{v}=\partial R^{2} L_{v}
$$

Se utiliza el valor verdadero de una cantidad denominado $x$ y el valor medido, inferido o aproximado es $x_{0}$.

Entonces, el error relativo se define por:

$\delta x=\frac{\Delta x}{x}=\frac{x_{0}-x}{x}$

Aquí $\Delta x$ es el error absoluto. El porcentaje de error es cien multiplicado por el error relativo para obtener el porcentaje.

El error relativo bajo estudio se calcula como $\left(E_{v}\right.$ aproximado $-E_{v}$ real)/Ev real. Se multiplica por cien para obtener el porcentaje. Se considera $E_{v}$ real como el valor convencionalmente verdadero y Ev aprox. como un valor medido que tiene errores en su medición, por eso es aproximado. Al realizar el desarrollo de las ecuaciones para el error relativo se obtiene que sea igual a $R^{2} / D^{2}$. Este criterio primario es el radio de la fuente al cuadrado entre la distancia al cuadrado. Esta es la base para obtener el criterio de la dimensión de la última columna. Un porcentaje de error de $1 \%$ relaciona una distancia $D$ de $0,15 \mathrm{~m}$ con un radio de 0,015 . Pero esto no es correcto.

El criterio de la dimensión de la sección, analizado en el otro artículo, establece que se debe comparar la dimensión máxima, en este caso el diámetro con la distancia. Por esto la relación obtenida de radio $(R)$ debe pasarse a diámetro $(2 R)$. Esto es $R^{2}$ se convierte en $4 R^{2}$. Por eso la columna de porcentaje de error se multiplica por cuatro para obtener el criterio de la dimensión.

Ahora, el porcentaje de error del $1 \%$ corresponde a la columna resaltada, donde la distancia es $0,3 \mathrm{~m}$ que es diez veces el diámetro de $0,03 \mathrm{~m}$ ( $3 \mathrm{~cm}$. de diámetro) del ejemplo en desarrollo. Entonces, para que el porcentaje de error (relativo) entre la iluminancia real y la iluminancia aproximada (con ley de cuadrado inverso) sea menor a $1 \%$, la razón entre $D$ a $2 R$ (máxima dimensión lateral) debe ser 10:1 ya mencionada. Por el criterio de la dimensión se obtiene:

$d x($ dimensión lateral máxima $)=2 R=0,03 \mathrm{~m}=$ $3,0 \mathrm{~cm}$.

$D$ (distancia entre fuente $\mathrm{y}$ detector $)=0,3 \mathrm{~m}=$ $30 \mathrm{~cm}$.

Para una fuente de luz con dimensión de $3 \mathrm{~cm}$ de diámetro, después de $30 \mathrm{~cm}$ las mediciones son más exactas, para efectos de obtener una ley de cuadrado inverso y relacionar la iluminancia y la intensidad apropiadamente.

Se termina el análisis con un gráfico que presenta el criterio de la dimensión en otra forma coherente. Se utiliza para comparación más lógica, la relación distancia al cuadrado al diámetro al cuadrado $D^{2} /(2 R)^{2}$ pero no en porcentaje, solo su razón.

Se presentan los valores $E_{v}$ aprox (segunda columna, Cuadro 2), excitancia luminosa $M_{v}$ (tercera columna, Cuadro 2) que corresponde al caso de una fuente lambertiana (perfecta) y $E_{v}$ real (cuarta columna, Cuadro 2).

Los porcentajes de error de las columnas cinco y seis se observan en el gráfico como separaciones entre las curvas.

La relación $D^{2} /(2 R)^{2}=100$ corresponde al valor buscado, esto porque $2 R=0,1 D$. Al hacer la razón $D^{2} /(2 R)^{2}=\left(1 / 0,1^{2}\right)$ que es igual a 100 . Nuevamente se indica que es el porcentaje de error de $0,25 \%$ que al multiplicar por 4 da el resultado de $1 \%$ objetivo. Esta es la forma de obtener el criterio de la dimensión. Con los datos del Cuadro 2 se obtiene el gráfico de errores de la Figura 1.

En el eje $X$ se encuentra que cuando $D^{2} / R^{2}$ es 100 , el error relativo de la ley de cuadrado inverso es $0,25 \%$, el valor descrito anteriormente. Se notan además las tendencias de las curvas. A distancias largas $D$ la curva $E_{v}$ aproximadamente se une con la curva $E_{v}$ real. Igualmente, a distancias cortas la curva de excitancia luminosa $M_{v}$ se une con la curva $E_{v}$ real. Esto indica que la fuente se comporta como cercana a lambertiana (emisor perfecto) porque a cortas distancias toda la luz se emite pareja; es una fuente cercana y la iluminancia no varía con la distancia. Esto ocurre cuando 


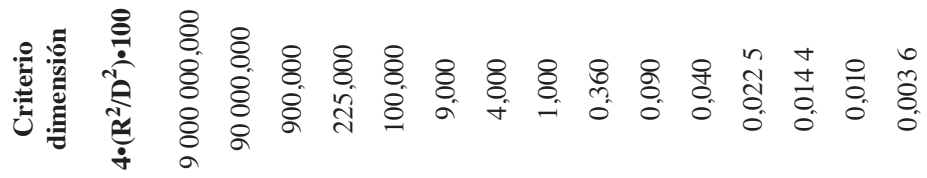

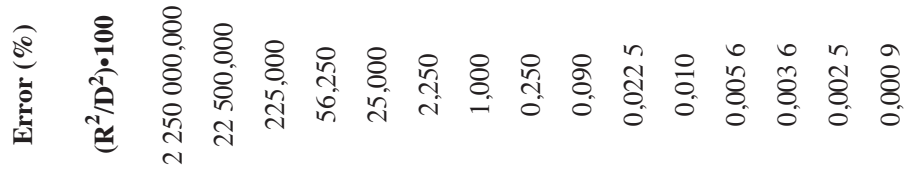

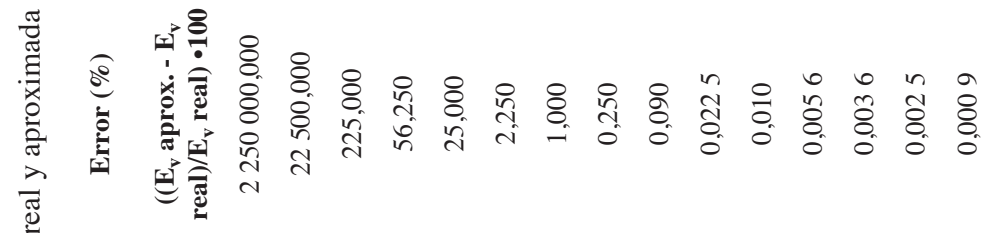

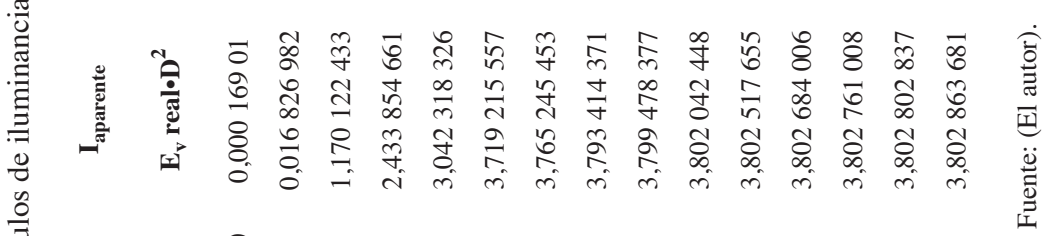

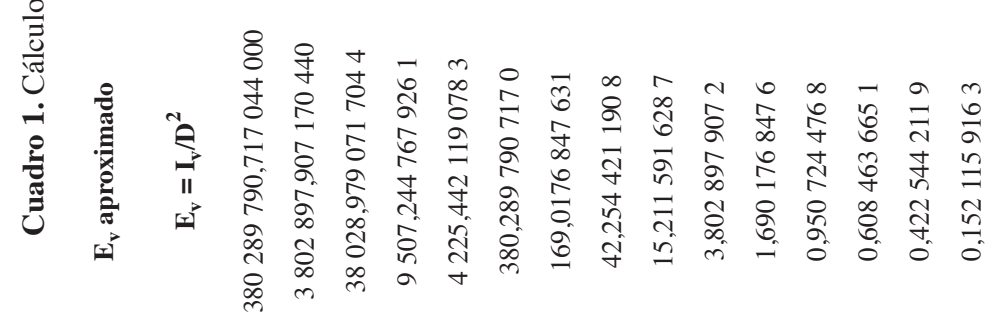

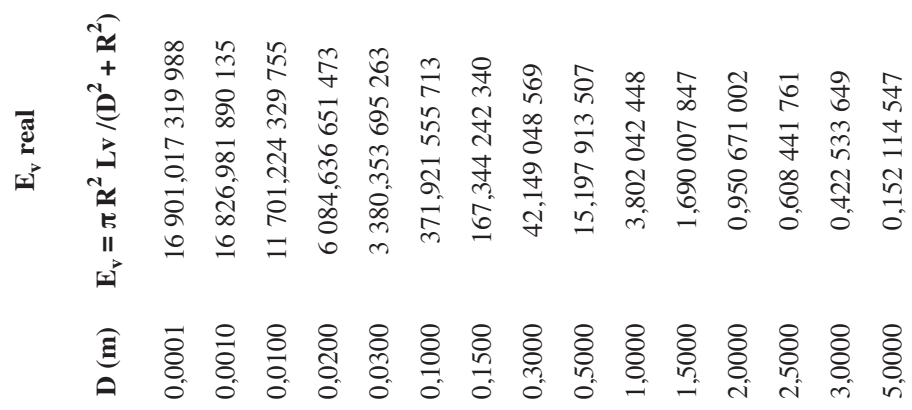




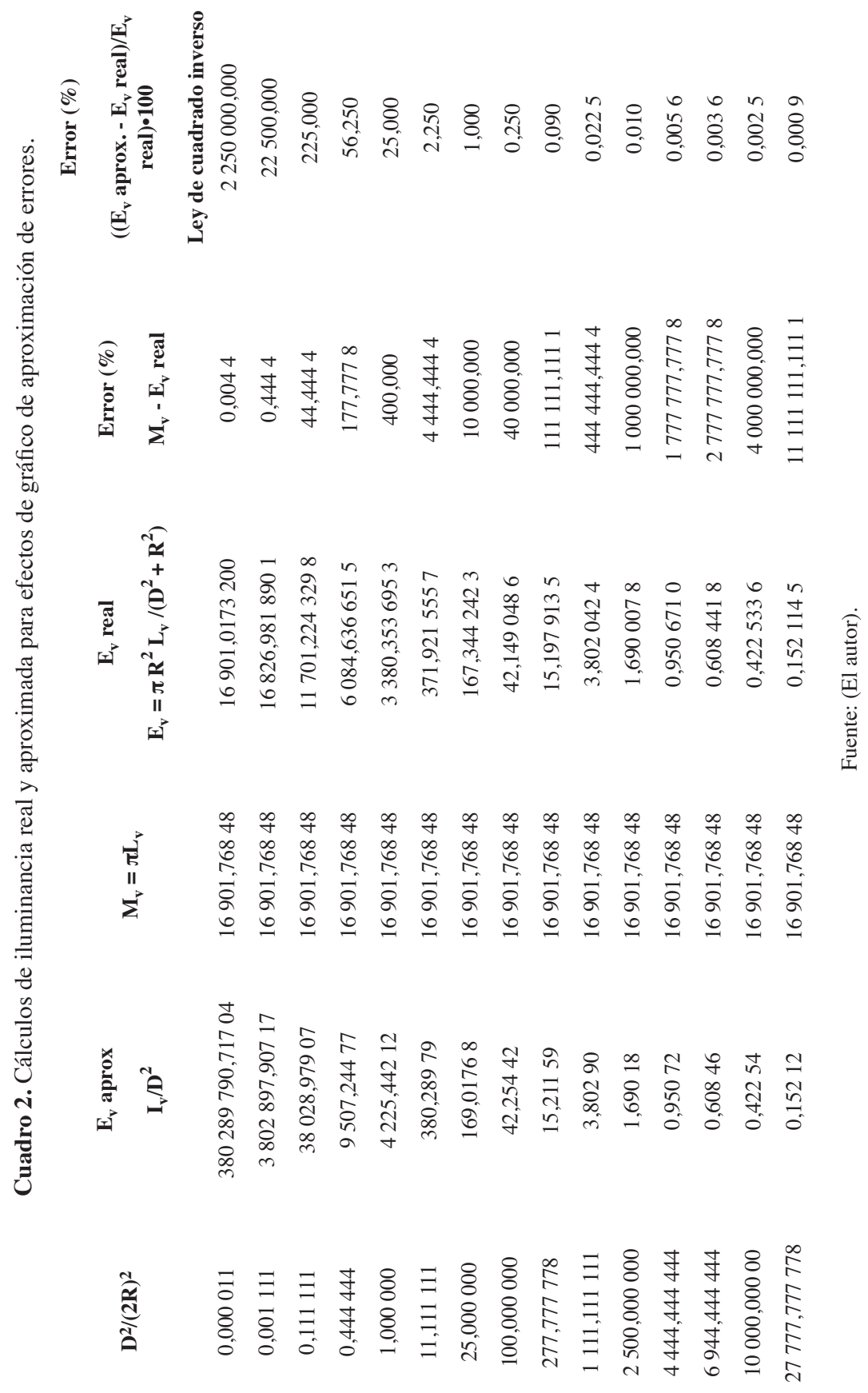




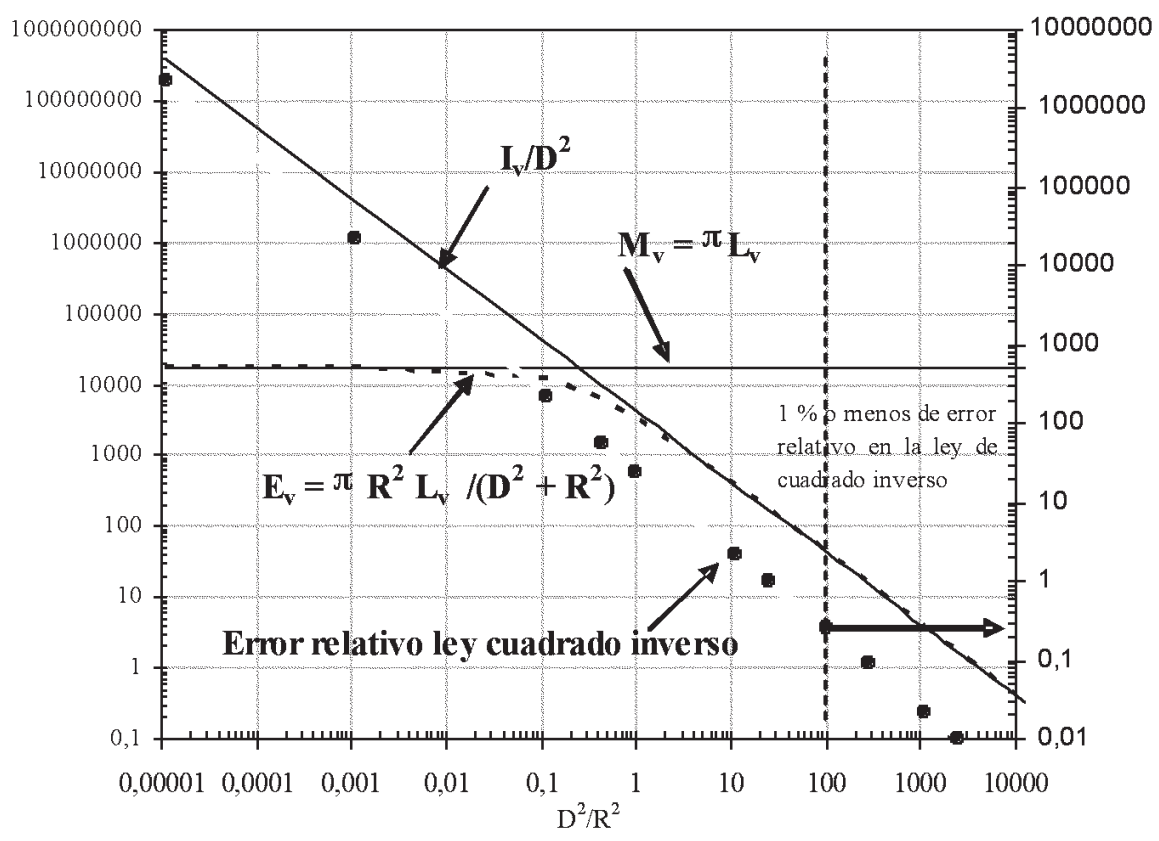

Figura 1. Errores en fuente puntual y consideración de la ley de cuadrado inverso.

Fuente: (Marín, 2008).

$D^{2} / R^{2}$ es menor que 0,01 , si se considera en porcentaje de nuevo el $1 \%$.

Entonces para no incurrir en errores, tanto de aproximación de la ley de cuadrado inverso, así como de tomar una fuente real como isotrópica (lambertiana), se deben considerar distancias de al menos diez veces la dimensión máxima de la fuente. Aunque no se puede generalizar a otras formas de fuentes de luz a partir de este ejemplo, el comportamiento es muy similar a la distribución de luminancia si la fuente es cercanamente lambertiana.

Se puede medir apropiadamente en el banco fotométrico la intensidad, para calibrar medidores de iluminación a partir de una lámpara de intensidad patrón conocida. Si se cuenta con una fuente de luz lambertiana en forma de disco se puede montar en un banco fotométrico y medir la intensidad desde "lejos" utilizando un medidor de iluminancia y obtener valores de $E_{v}$ real como en el Cuadro 1 (si la fuente de luz fuera similar).
Luego se calcula la columna de $I_{\text {aparente }}$ del Cuadro 1 al multiplicar cada valor $E_{v}$ real por $D_{2}$, como se hace en la ecuación (2) de $E_{v}$ aproximada para la fuente entera. Al incrementar $D$ se nota que la intensidad aparente alcanza la intensidad real de la fuente entera desde muy lejos. En notación de límite se tiene:

$I_{v}=\lim _{D \rightarrow \infty} E_{v} D^{2}$

Se llega al mismo resultado desde la ecuación (1). Para $D>>R$ :

$E_{v}=\frac{\partial R^{2} L_{v}}{D^{2}}=\frac{L_{v} A_{\text {proyectada dela fuente }}}{D^{2}}=\frac{I_{v}}{D^{2}}$

Así, si se desea realizar una práctica experimental donde se mida la iluminancia, con 
condiciones geométricas conocidas, a diferentes distancias, se puede calcular la fuente puntual virtual, entre otros, se puede obtener la intensidad, la luminancia y el flujo radiante.

\section{PRÁCTICA EXPERIMENTAL PARA LA MEDICIÓN DE ILUMINANCIA Y LA LEY DE CUADRADO INVERSO CON FUENTES DE LUZ REALES}

Si el banco fotométrico reúne las condiciones como mantener el sensor de un medidor de iluminancia perpendicular a la fuente de luz en un punto y considerar que la fuente es cercanamente lambertiana con forma de disco a través de una apertura, se puede aplicar una medición exacta de la iluminancia. En el análisis de problemas ópticos se supone que la luz proviene de una fuente puntual idealizada.

La Figura 1 muestra un gráfico representativo de una fuente puntual y el comportamiento de la luz emitida, con base en los cuadros aplicados al análisis de errores relativos en el cálculo de la iluminancia real y aproximada y la ley de cuadrado inverso.

Se asume que la fuente emite igualmente en todas las direcciones, que la luz viaja a la misma velocidad en todas las direcciones y se esparce en un patrón esférico. La intensidad luminosa $I_{v 0}$ se distribuye sobre la superficie de este patrón esférico conforme se aleja desde la fuente.

Si se supone que la luz intercepta una unidad de área $A$, a una distancia $r$ desde la fuente, la intensidad de la luz en esta unidad $I_{v}$ es:

$I_{v}=\frac{I_{v 0} A}{A^{T}}$

Aquí $A^{T}$ es el área de la superficie total de la esfera de radio $r$. Así el área de la superficie de la esfera es $A^{T}=4 \pi r^{2}$. La intensidad interceptada es:

$I_{v}=\frac{I_{v 0} A}{4 \partial r^{2}}$

Para un área interceptada dada, la intensidad recibida decrece desde una fuente puntual proporcionalmente con $\mathrm{r}^{-2}$. Radiación desde una fuente puntual es la ley de radiación de inverso del cuadrado de la distancia. Si se usan espejos para concentrar la luz desde la fuente, la ecuación es:

$I_{v}=\frac{I_{v 0} K A}{4 \partial r^{2}}$

Aquí $K$ es el factor de concentración. Por ejemplo, si la luz desde la fuente se concentra totalmente en una mitad de la esfera $K$, es igual a dos. Si la luz cubre solo un octavo de la esfera $K$ es igual ocho.

La siguiente práctica experimental de laboratorio se realiza en el banco fotométrico como parte de los objetivos de este proyecto de investigación. Se utiliza el siguiente equipo:

- Un banco fotométrico

- Una fuente de luz blanca colimada con un sistema óptico Fostec EKE.

- Un mecanismo de desplazamiento optomecánico.

- Un sistema de detección GigaHertz Optik modelo P9710.

- Pantalla de observación, regla para óptica, lámina con apertura de $1 \mathrm{~mm}$.

Se debe recalcar que no se afecta la relación decuadrado inverso porsimplemente concentrar la luz. De hecho, si la luz es divergente desde la fuente del todo, o sea, si el haz tiene forma de cono, no importando qué tan pequeño sea el ángulo del cono, la ley de cuadrado inverso se aplica. La mayoría de fuentes de iluminación no son fuentes puntuales, pero si se mantiene el tamaño efectivo de la fuente lo suficientemente pequeño respecto a la distancia, se puede considerar como una fuente puntual.

Se utiliza el siguiente procedimiento:

1. Montar el banco fotométrico como se muestra en la Figura 2.

2. Utilizar un agujero de $1 \mathrm{~mm}$ de diámetro perforado en una lámina de aluminio.

3. Colocar la lámina de aluminio en el centro del haz de la fuente de luz a 50 mm, Figura 2.

4. Mover la pantalla de observación a unos 50 mm de la lámina de aluminio. 


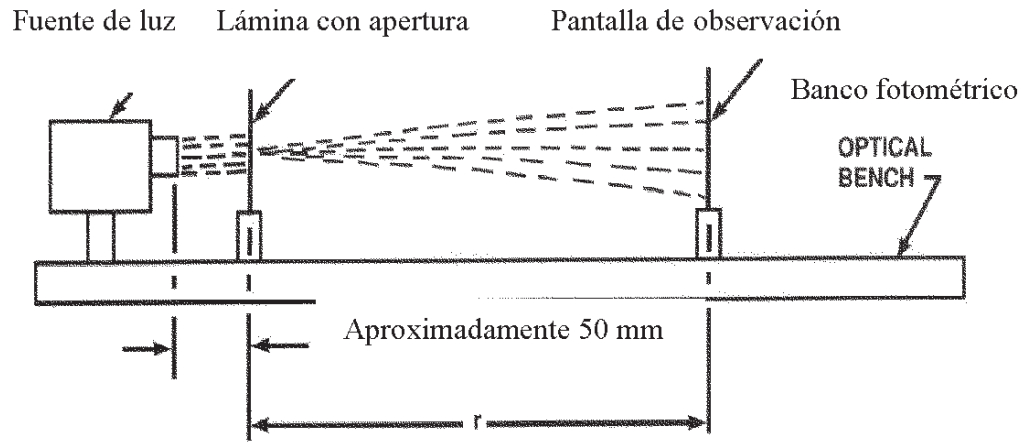

Figura 2. Montaje Experimental.

Fuente: (Marín, 2008).

5. Se debe ver un círculo de luz en la pantalla.

6. Medir con la mejor exactitud el diámetro (d) en milímetros del círculo de luz sobre la pantalla.

7. Anotar el espaciado $(r)$ entre la lámina de aluminio y la pantalla de observación.

8. Incrementar el espaciado en tramos de $50 \mathrm{~mm}$.

9. Repetir los pasos 6 y 7.

10. Repetir los pasos 8 y 9 hasta obtener diez mediciones.

11. Calcular el área (A) del círculo de luz para cada juego de datos con $A=\pi d^{2} / 4$.

12. Elevar al cuadrado los datos de espaciado $\left(r^{2}\right)$. Anotar en un cuadro.
13. Graficar una curva de área $(A)$ versus el cuadrado del espaciado $r^{2}$.

14. Mantener la lámina de $1 \mathrm{~mm}$ de diámetro y sustituir la pantalla de observación por el detector de un medidor de iluminancia. Realizar tres mediciones de valores de iluminancia a las mismas distancias. Anotar en un cuadro.

15. Calcular la intensidad luminosa y graficar una curva de iluminancia versus el cuadrado del espaciado $r^{2}$.

16. Analizar los resultados buscando la confirmación de ley de cuadrado inverso con base en las ecuaciones y considerando

Cuadro 3. Datos para el cálculo de la Ley del Cuadrado Inverso para iluminancia.

$\begin{array}{cccccc} & d & d & r & A & r^{2} \\ \text { Medición } & (\mathbf{m m}) & (\mathbf{m}) & (\mathbf{m m}) & \left(\mathbf{m m}^{2}\right) & \left.\mathbf{( m}^{\mathbf{2}}\right) \\ 1 & 2,0 & 0,0020 & 50 & 3,142 & 0,0025 \\ 2 & 2,2 & 0,0022 & 100 & 4,524 & 0,0100 \\ 3 & 3,0 & 0,0030 & 150 & 7,069 & 0,0225 \\ 4 & 3,4 & 0,0034 & 200 & 8,042 & 0,0400 \\ 5 & 4,0 & 0,0040 & 250 & 12,566 & 0,0625 \\ 6 & 5,0 & 0,0050 & 300 & 19,635 & 0,0900 \\ 7 & 5,2 & 0,0052 & 350 & 21,237 & 0,1225 \\ 8 & 6,0 & 0,0060 & 400 & 30,191 & 0,1600 \\ 9 & 7,0 & 0,0070 & 450 & 38,485 & 0,2025 \\ 10 & 8,0 & 0,0080 & 500 & 50,265 & 0,2500\end{array}$




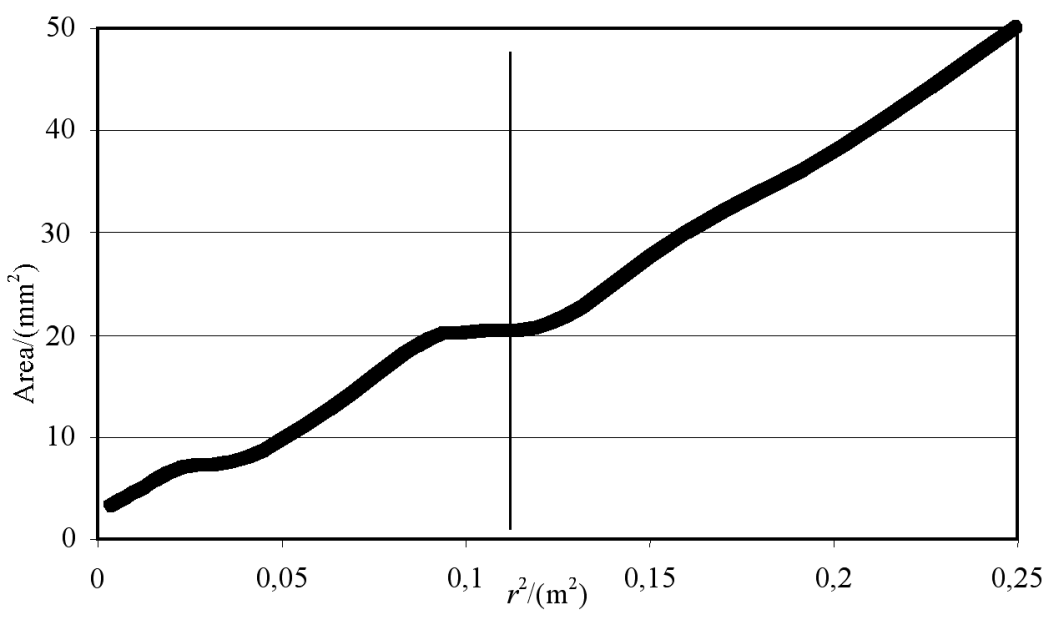

Figura 3. Variación del área con la distancia al cuadrado.

Fuente: (Marín, 2008).

que todo se ha mantenido constante, excepto $A$ y $r^{2}$.

17. Analizar por qué el sistema utilizado actúa como una fuente puntal. Explicar el resultado de la ley de cuadrado inverso con base en mediciones de iluminancia y relacionar la distancia a las dimensiones de la fuente con los valores de intensidad luminosa.

\section{ANÁLISIS DE RESULTADOS}

La fuente de luz utilizada es una lámpara incandescente de halógeno de $150 \mathrm{~W}$. Tiene una apertura de salida de $17,5 \mathrm{~mm}$ como obstrucción pero se pasa por un agujero de $1 \mathrm{~mm}$ de diámetro, que es el área emisora de luz por considerar.

Utilizando el criterio para tratar una fuente como fuente puntual, se debe obtener el producto de las dimensiones laterales y ser menor al cuadrado de

Cuadro 4. Mediciones de iluminancia, intensidad luminosa y ley de cuadrado inverso para la iluminancia.

\begin{tabular}{|c|c|c|c|c|c|c|}
\hline $\begin{array}{c}\mathbf{r} \\
(\mathrm{mm})\end{array}$ & $\begin{array}{l}\text { 1. } \mathrm{E}_{\mathrm{v}} \\
(\mathbf{l x})\end{array}$ & $\begin{array}{l}\text { 2. } \mathrm{E}_{\mathrm{v}} \\
(\mathbf{l} \mathbf{x})\end{array}$ & $\begin{array}{r}\text { 3. } \mathrm{E}_{\mathrm{v}} \\
\text { (lx) }\end{array}$ & $\begin{array}{c}\text { Promedio } \mathbf{E}_{\mathbf{v}} \\
\text { (lx) }\end{array}$ & $\begin{array}{c}\mathbf{r}^{2} \\
\left(\mathbf{m}^{2}\right)\end{array}$ & $\begin{array}{c}I_{v} \\
(c d)\end{array}$ \\
\hline 50,0 & 45,0 & 47,8 & 44,5 & 45,767 & 0,0025 & 0,114 \\
\hline 100 & 58,5 & 61,2 & 59,0 & 59,567 & 0,0100 & 5,957 \\
\hline 150 & 73,6 & 67,5 & 65,8 & 68,967 & 0,0225 & 1,552 \\
\hline 200 & 78,1 & 81,4 & 78,8 & 79,433 & 0,0400 & 3,177 \\
\hline 250 & 73,6 & 74,5 & 75,8 & 74,633 & 0,0625 & 4,665 \\
\hline 300 & 65,3 & 67,3 & 67,0 & 66,533 & 0,0900 & 5,988 \\
\hline 350 & 60,8 & 66,4 & 61,1 & 62,767 & 0,1225 & 7,689 \\
\hline 400 & 56,3 & 62,9 & 57,6 & 58,933 & 0,1600 & 9,429 \\
\hline 450 & 50,0 & 51,8 & 52,9 & 51,567 & 0,2025 & 10,442 \\
\hline 500 & 45,0 & 47,5 & 48,4 & 46,967 & 0,2500 & 11,742 \\
\hline
\end{tabular}

Fuente: (El autor, 2008) 


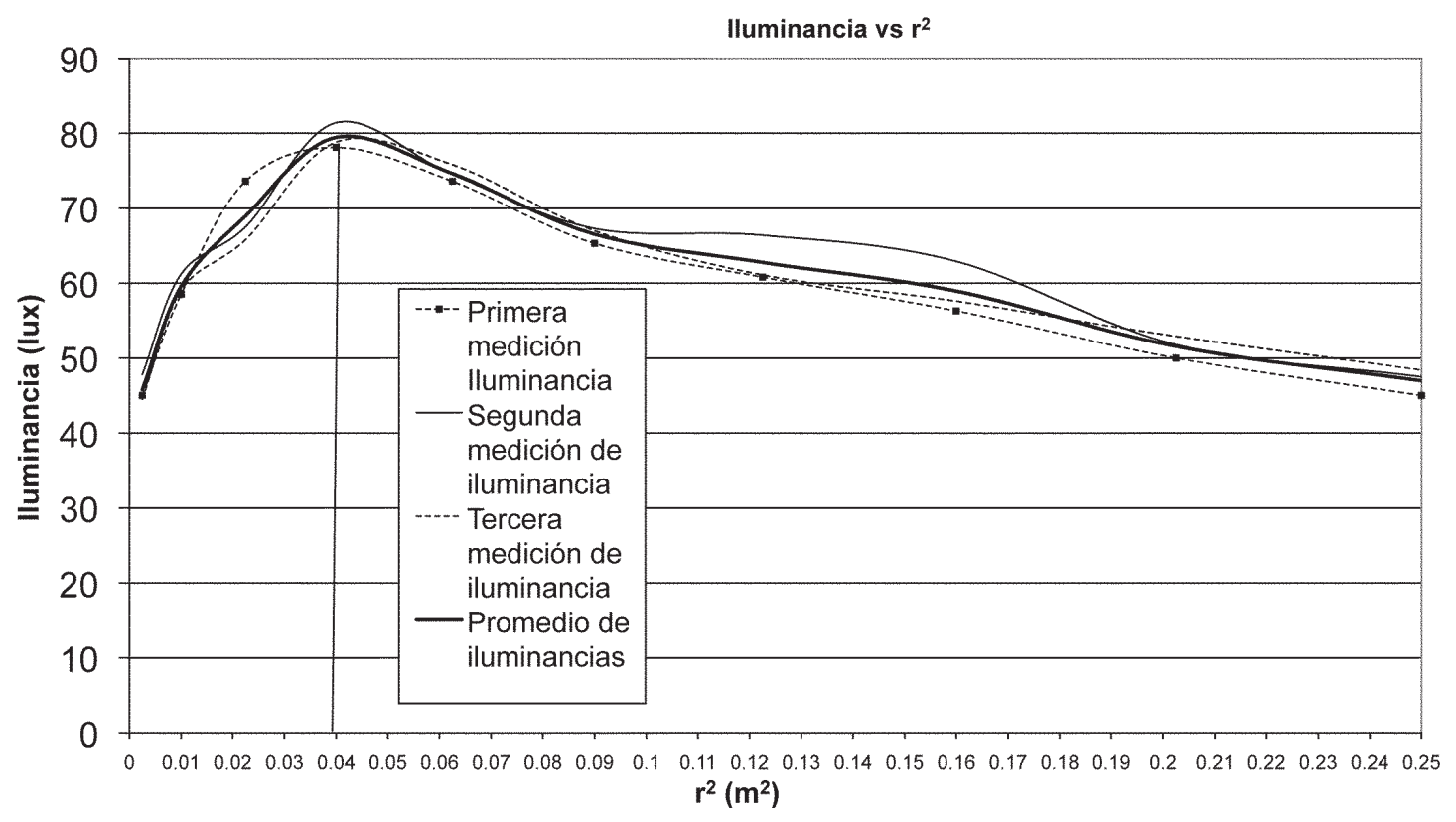

Figura 4. Variación de la iluminancia con la distancia al cuadrado.

Fuente: (Marín, 2008).

la distancia a la fuente: $d x \cdot d y<<r^{2}$, esto es $1 \mathrm{~mm}$ $\times 1 \mathrm{~mm}=1 \mathrm{~mm}^{2}$. Se despeja $r$ como $1 \mathrm{~mm}^{2} / 0,01=$ $r^{2}$. Entonces $r$ debe ser mayor a $10 \mathrm{~mm}(1 \mathrm{~cm})$ para considerar la ley de cuadrado inverso. Se realiza el Cuadro 3 para la ley de cuadrado inverso teórico.

Al graficar la variación del área con la distancia al cuadrado en la Figura 3, con los datos del cuadro 3 , se observa en la marca que $r^{2}$ es $0,115 \mathrm{~m}^{2}$, lo que implica que $r$ es 339 $\mathrm{mm}(39 \mathrm{~cm})$ para obtener una respuesta lineal apropiada. La marca indica un punto en la gráfica, no se presentan variaciones no lineales y cambios de pendiente.

Se cumple que $r$ debe ser mayor a $10 \mathrm{~mm}$ de acuerdo con el criterio y se confirma el criterio con el valor de $\mathrm{r}$ adquirido de la gráfica pero hasta $339 \mathrm{~mm}$, lo que permite considerar que se requiere una distancia grande como parámetro en mediciones fotométricas.

Al graficar la variación de la iluminancia con la distancia al cuadrado en la Figura 4, con los datos del cuadro 4 , se observa en la marca que

Cuadro 5. Datos para mediciones de iluminancia, intensidad luminosa y la ley de cuadrado inverso para la iluminancia con agujero de $1 \mathrm{~mm}$, a otras distancias a partir de $r=500 \mathrm{~m}$.

\begin{tabular}{|c|c|c|c|c|c|c|}
\hline $\begin{array}{c}\mathbf{r} \\
(\mathrm{mm})\end{array}$ & $\begin{array}{r}\text { 1. Ev } \\
(\mathbf{l x})\end{array}$ & $\begin{array}{r}\text { 2. } E_{v} \\
\text { (lx) }\end{array}$ & $\begin{array}{r}\text { 3. } \mathrm{E}_{\mathrm{v}} \\
\text { (lx) }\end{array}$ & $\begin{array}{c}\text { Promedio } \mathrm{E}_{\mathbf{v}} \\
\text { (lx) }\end{array}$ & $\begin{array}{c}\mathbf{r}^{2} \\
\left(\mathbf{m}^{2}\right)\end{array}$ & $\begin{array}{c}\mathbf{I}_{\mathbf{v}} \\
(\mathbf{c d})\end{array}$ \\
\hline 500 & 47,0 & 47,0 & 47,0 & 47,0 & 0,2500 & 11,75 \\
\hline 550 & 41,5 & 41,5 & 41,5 & 41,5 & 0,3025 & 12,55 \\
\hline 600 & 36,1 & 36,1 & 36,1 & 36,1 & 0,3600 & 12,99 \\
\hline 650 & 33,0 & 33,0 & 33,0 & 33,0 & 0,4225 & 13,94 \\
\hline 700 & 30,5 & 30,5 & 30,5 & 30,5 & 0,4900 & 14,94 \\
\hline
\end{tabular}

Fuente: (El autor, 2008). 


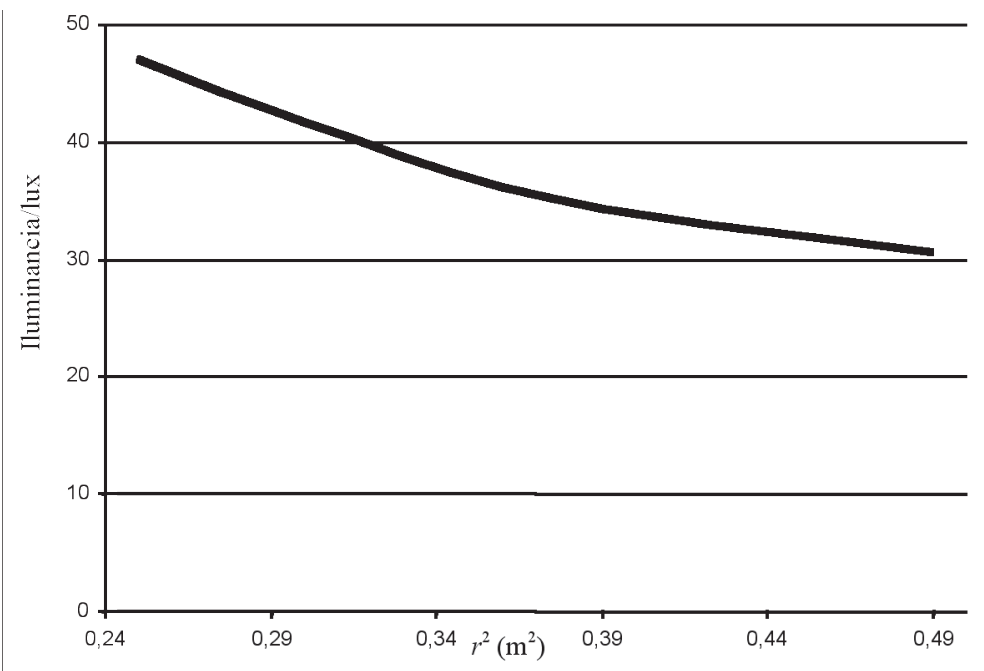

Figura 5. Variación de la iluminancia con la distancia al cuadrado.

Fuente: (Marín, 2008).

$r^{2}$ es $0,045 \mathrm{~m}^{2}$, lo que implica que $r$ es $212 \mathrm{~mm}$ $(21,2 \mathrm{~cm})$ para obtener una respuesta apropiada de decaimiento con ley de cuadrado inverso.

Se debe cumplir que $r$ debe ser mayor a $10 \mathrm{~mm}$ para acordar el criterio y se confirma con el valor de $r$ adquirido de la gráfica que se puede utilizar en mediciones fotométricas de iluminancia. Como resumen, $r$ debe ser mayor a $10 \mathrm{~mm} \mathrm{y}$ se obtiene en las mediciones de área un valor para $r$ de más de $339 \mathrm{~mm}$ y en las mediciones de iluminancia un valor para $r$ de más de 212 $\mathrm{mm}$. Esto hace que concuerde la teoría con los valores de la práctica experimental. En cuanto al valor de la intensidad, el detector del medidor de iluminancia tiene un diámetro de $7 \mathrm{~mm}$, por lo que las mediciones con un área proyectada después de la apertura de $1 \mathrm{~mm}$ menores de ese diámetro no se ajustan a una fuente divergente

Cuadro 6. Iluminancia e intensidad luminosa sin agujero a distancias desde $r=100 \mathrm{~m}$ hasta $r=1200 \mathrm{~m}$.

$\begin{array}{cccc}r & E_{v} & \mathbf{r}^{2} & I_{v} \\ (\mathbf{m m}) & (\mathbf{l x}) & \left(\mathbf{m}^{2}\right) & (\mathbf{c d}) \\ 100 & 2379 & 0,01 & 23,79 \\ 200 & 336 & 0,04 & 13,44 \\ 300 & 120 & 0,09 & 10,8 \\ 400 & 57 & 0,16 & 9,12 \\ 500 & 34 & 0,25 & 8,5 \\ 600 & 22 & 0,36 & 7,92 \\ 700 & 16 & 0,49 & 7,84 \\ 800 & 12,1 & 0,64 & 7,74 \\ 900 & 9,5 & 0,81 & 7,695 \\ 1000 & 7,5 & 1 & 7.50 \\ 1100 & 6,1 & 1,21 & 7,38 \\ 1200 & 5,1 & 1,44 & 7,34\end{array}$

Fuente: (El autor, 2008). 


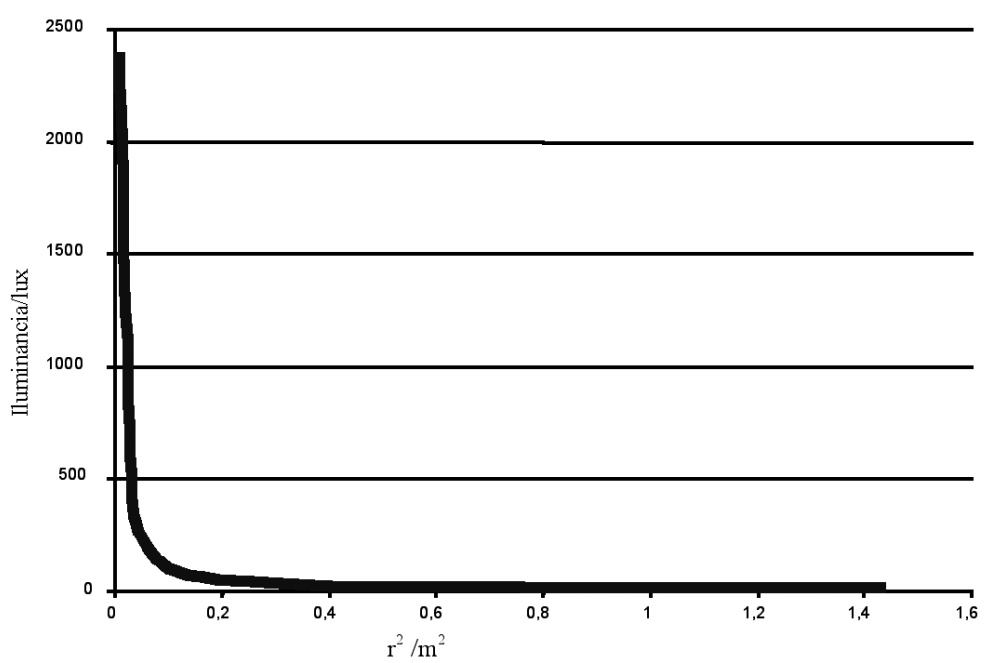

Figura 6. Variación de la iluminancia con la distancia al cuadrado.

Fuente: (Marín, 2008).

Cuadro 7. Iluminancia con intensidad luminosa teórica y ley de cuadrado inverso desde $r=1 \mathrm{~m}$ a $10 \mathrm{~m}$

$\begin{array}{cccc}\begin{array}{c}\boldsymbol{I}_{v} \\ (\mathbf{c d})\end{array} & \begin{array}{c}\boldsymbol{r} \\ (\mathbf{m})\end{array} & \begin{array}{c}\boldsymbol{r}^{\mathbf{2}} \\ \left(\mathbf{m}^{\mathbf{2}}\right)\end{array} & \begin{array}{c}\boldsymbol{E}_{v} \\ (\mathbf{l x})\end{array} \\ 100 & 1 & 1 & \\ 100 & 2 & 4 & 100 \\ 100 & 3 & 9 & 25 \\ 100 & 4 & 16 & 11,11111111 \\ 100 & 5 & 25 & 6,25 \\ 100 & 6 & 36 & 4 \\ 100 & 7 & 49 & 2,777777778816327 \\ 100 & 8 & 64 & 1,5625 \\ 100 & 9 & 81 & 1,234567901 \\ 100 & 10 & 100 & 1\end{array}$

Fuente: (El autor, 2008).

desde una fuente puntual, sino colimada aun por el sistema de lentes. En las mediciones con valores 10,442 cd y 11,742 cd se observa un error bajo, considerando que a pesar de cumplirse la ley de cuadrado inverso, no hay divergencia suficiente. Para probar esto se realizaron dos mediciones adicionales, una con el mismo montaje anterior a mayores distancias, partiendo de la medición de $500 \mathrm{~mm}$ y otra con la fuente directa hacia el detector, sin el agujero de $1 \mathrm{~mm}$ y se obtuvo:
Al graficar los datos en la Figura 5, con los datos del Cuadro 5, se observa a partir de $r^{2}=$ 0,25 hasta $r^{2}=0,49$, lo que implica que desde $r$ $=500 \mathrm{~mm}(0,5 \mathrm{~m})$ hasta $r=700 \mathrm{~mm}(0,7 \mathrm{~m})$ para obtener una respuesta apropiada de decaimiento con ley de cuadrado inverso.

Se observa un decaimiento monótono esperado con $r\left(y r^{2}\right)$, que confirma que a largas distancias de la fuente se cumple la ley de cuadrado inverso. Los valores de intensidad tienden a estabilizarse 


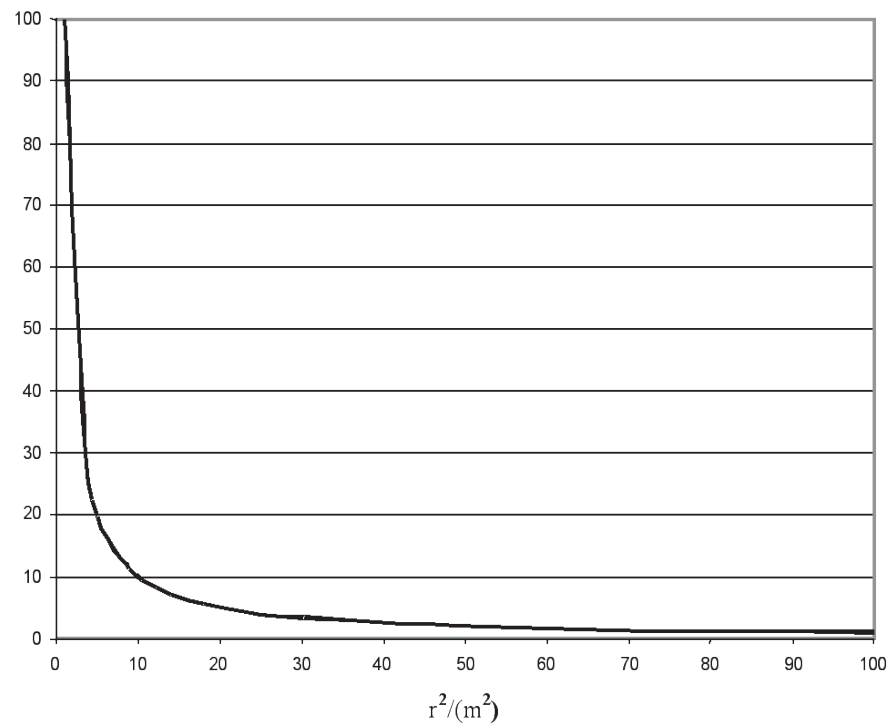

Figura 7. Variación de la iluminancia teórica con la distancia al cuadrado desde $1 \mathrm{~m}$ a $10 \mathrm{~m}$.

Fuente: (Marín, 2008).

Fuente de luz (Fostec)

Sistema optomecánico y banco fotométrico

Detector (GigaHertz Optik)

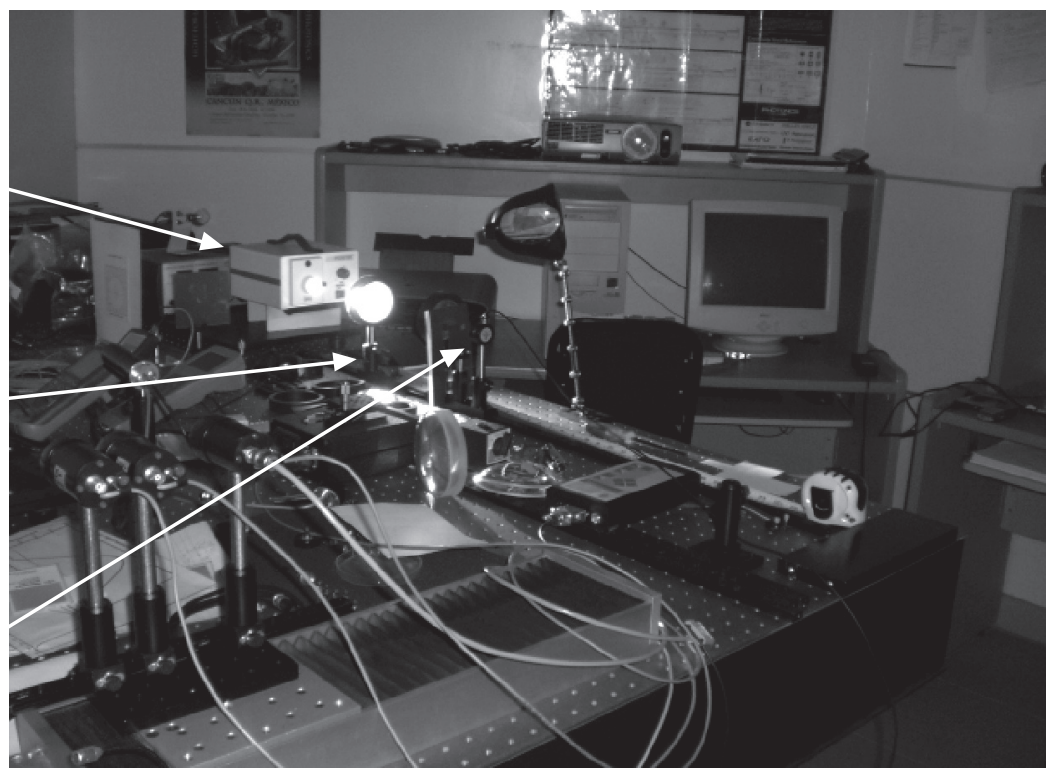

Figura 8. Vista general del montaje experimental básico del banco fotométrico en el LAFTLA (Laboratorio de Fotónica y Tecnología Láser).

Fuente: (El autor, 2008). 


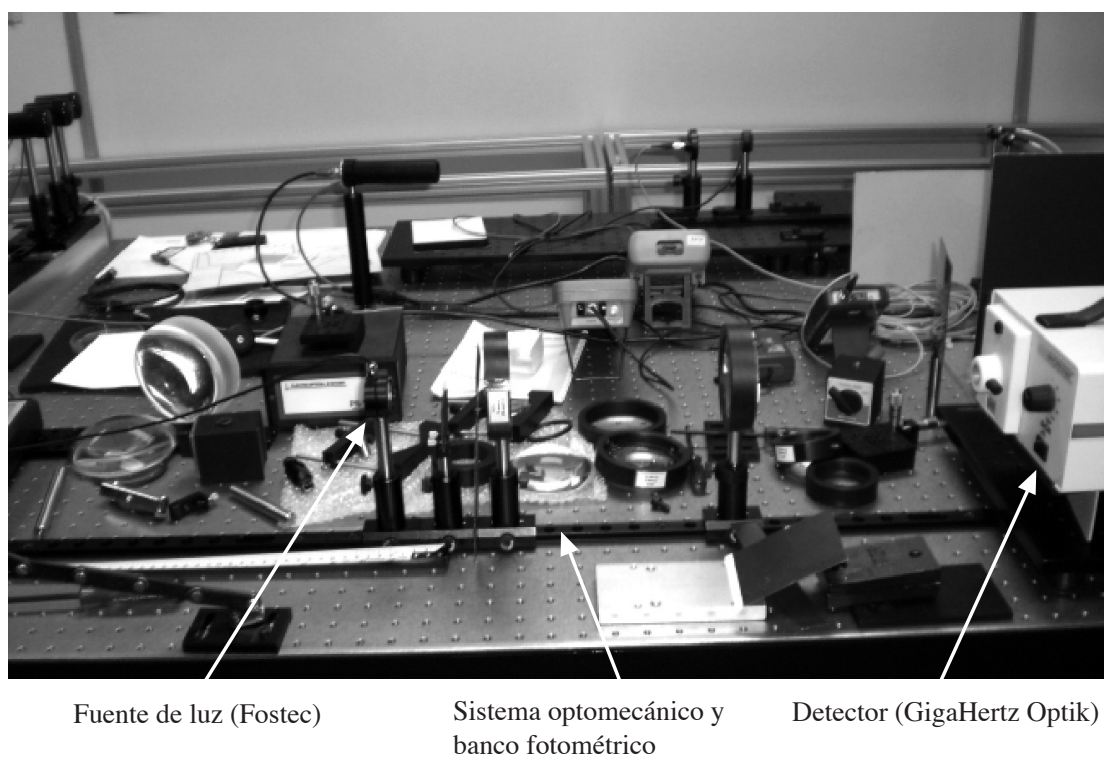

Figura 9. Detalles del montaje experimental básico del banco fotométrico en el LAFTLA (Laboratorio de Fotónica y Tecnología Láser).

Fuente: (El autor, 2008).

y se logra un valor que se uniformiza a muy largas distancias. Utilizando el criterio de fuente puntual se obtiene el producto como $d x \cdot d y<<$ $r^{2}$, o sea $17,50 \mathrm{~mm} \times 17,50 \mathrm{~mm}=306,25 \mathrm{~mm}^{2}$. Se despeja $r$ como $306,25 \mathrm{~mm}^{2} / 0,01=r^{2}$. Así $r$ debe ser mayor a $175 \mathrm{~mm}$ para considerar la ley de cuadrado inverso.

Se grafican los datos del Cuadro 6 en la Figura 6 y se nota a partir de $r^{2}=0,10$ aproximadamente una respuesta apropiada de decaimiento con ley de cuadrado inverso, donde $r=0,316 \mathrm{~m}$ o sea $316 \mathrm{~mm}$.

Se observa el decaimiento monótono con $r\left(y r^{2}\right)$ que confirma que a largas distancias de la fuente se cumple la ley de cuadrado inverso. La distancia de $316 \mathrm{~mm}$ concuerda con que a una distancia mayor a $175 \mathrm{~mm}$, la curva se estabiliza suavemente. Para efectos de verificar este caso de una fuente de luz extendida, se considera la variación teórica de una intensidad de $100 c d$ y la iluminancia resultante a varias distancias según el Cuadro 7.

Al graficar estos datos en la Figura 7, con los datos de la Cuadro 7, se observa que a partir de $r^{2}=015$, o sea aproximadamente $387 \mathrm{~mm}$, se obtiene una respuesta apropiada de decaimiento con la ley de cuadrado inverso. La forma concuerda con el valor obtenido de la Figura 6 en el comportamiento general de la ley de cuadrado inverso.

Por ello las mediciones del Cuadro 5 son correctas y se obtiene que el comportamiento de la ley de cuadrado inverso para la iluminancia se cumple cuando la distancia cumple con el requisito de ser larga comparada con las dimensiones de la apertura.

La Figura 8 muestra una vista con luz ambiente moderada para efectos de la foto, donde se observa el montaje general del banco fotométrico sobre la mesa óptica siguiendo el esquema de la Figura 8 y detalles en la Figura 9.

\section{CONCLUSIONES}

1. La ley de cuadrado inverso para la iluminancia cumple con el criterio de las dimensiones, tanto para aberturas pequeñas como para grandes, según se logró determinar con 
las mediciones descritas. Así que para mediciones de (irradiancia) iluminancia y su relación con la intensidad, se deben tomar en cuenta mediciones a distancias mayores a las definidas en el criterio.

2. Todos los resultados del estudio y el análisis también permitieron revisar la teoría y comparar con los conocimientos existentes. Así, en futuras mediciones fotométricas se tendrá completamente claro cuál es la distancia de medición de magnitudes para calibración o ensayo, sin considerar efectos adversos de la difracción de Fresnel.

3. Los resultados del trabajo de investigación, además de publicarse para su divulgación, se utilizarán en cursos de grado y posgrado de la Carrera de Ingeniería Eléctrica en la Universidad de Costa Rica en temas de radiometría, fotometría, metrología de parámetros ópticos, teoría de campo electromagnético, mediciones ópticas de laboratorio y diseño de sistemas de iluminación, así como en cursos de extensión en el tema.

\section{REFERENCIA BIBLIOGRÁFICA}

Marín, L. (2008). Informe final proyecto de investigación. Determinación de la relación entre la difracción de Fresnel y la ley de cuadrado inverso en mediciones fotométricas. No. 322-A7-182, Vicerrectoría de Investigación, Universidad de Costa Rica. San José, Costa Rica.

\section{REFERENCIAS BIBLIOGRÁFICAS RECOMENDADAS}

DeCusatis, C. (Ed.). (1998). Handbook of applied photometry. New York: Optical Society of America (OSA).

Murdoch, J. (1994). Illumination engineering. New York: Visions Communications.

Lindsey, J. (1991). Applied illumination engineering. New Jersey: Prentice Hall.

\section{SOBRE EL AUTOR}

\section{Luis Diego Marín Naranjo}

Ingeniero eléctrico, M. Sc.

Coordinador del Laboratorio de Fotónica y Tecnología Láser

Escuela Ingeniería Eléctrica, Universidad de Costa Rica

San José, Costa Rica.

Teléfono: 8314-1672

Correo electrónico: 1marin@eie.ucr.ac.cr 